\title{
Synthesis, Characterization, and Thermal Kinetics of Mixed Gadolinium: Calcium Heptamolybdate System
}

\author{
R. K. Koul, Shivani Suri, Vishal Singh, and K. K. Bamzai \\ Crystal Growth \& Materials Research Laboratory, Department of Physics and Electronics, University of Jammu, Jammu 180006, India \\ Correspondence should be addressed to K. K. Bamzai; kkbamz@yahoo.com
}

Received 7 March 2014; Accepted 1 July 2014; Published 20 October 2014

Academic Editor: Alessandra Toncelli

Copyright ( 2014 R. K. Koul et al. This is an open access article distributed under the Creative Commons Attribution License, which permits unrestricted use, distribution, and reproduction in any medium, provided the original work is properly cited.

\begin{abstract}
Synthesis of mixed gadolinium calcium heptamolybdate (GdCaHM) system in silica gel medium using single gel single tube technique has been successfully achieved. The grown crystal exhibits various morphologies, which includes spherulites, multifaceted, and square platelets. The nature of the grown material was established by X-ray diffraction (XRD) studies. Fourier transform infrared spectroscopy (FTIR) study signifies the presence of heptamolybdate $\left(\mathrm{Mo}_{7} \mathrm{O}_{24}\right)$ and water symmetry structure, whereas energy dispersive X-ray analysis (EDAX) establishes the stoichiometric of the grown crystal as $\mathrm{GdCaMo}_{7} \mathrm{O}_{24} \cdot 8 \mathrm{H}_{2} \mathrm{O}$. The thermal behaviour was studied using the thermoanalytical techniques, which include thermogravimetry (TG), differential thermal analysis (DTA), and differential scanning calorimetry (DSC). Results obtained on the application of TG based models, namely, Horowitz-Metzger, Coats-Redfern, and Piloyan-Novikova, suggest the contracting cylindrical model as the relevant model for the thermal decomposition of the material. The kinetic parameters, namely, the order of reaction $(n)$, activation energy $\left(E_{a}\right)$, frequency factor $(Z)$, and entropy $\left(\Delta S^{*}\right)$, were also calculated using these three models.
\end{abstract}

\section{Introduction}

Rare earth (RE) compounds in general have been recognized to exhibit laser, piezoelectric, ferroelectric, ferroelastic, and fluorescent properties for a long time. RE molybdates having general formula $\mathrm{R}_{2}\left(\mathrm{MoO}_{4}\right)_{3}$ have been reported as a ferroelectric material [1]. The importance of these materials lies in their ferroelectric and ferroelastic properties finding immense application in electro- and acoustooptical devices [2-4]. RE heptamolybdate with general formula $\mathrm{R}_{2} \mathrm{Mo}_{7} \mathrm{O}_{24}$, belongs to the family of molybdates. Brixner [5] reported the growth of RE molybdates employing the Czochralski technique at elevated temperatures but the thermal stress introduced during the growth makes the crystal defective. In order to overcome these difficulties, it is worthwhile to grow some new RE molybdate crystals by other simple techniques. Large number of crystals has been grown at ambient temperature; therefore, growth at low temperature is expected to yield crystals with minimum defects. The crystal growth in gels at room temperature pioneered by Henisch and coworkers $[6,7]$ has been fully exploited in the investigation of growth and characterization of rare earth mixed crystals of Sm-Ba molybdate by Isac and Ittyachen [8]. Studies on the growth of pure lanthanum, neodymium, and mixed La-Nd heptamolybdate single crystals in silica gel have been carried out by Bhat et al. [913]. Growth, characterization, and thermal behaviour of pure Gd-heptamolybdate, mixed Gd-Ba molybdate, and Gd-Sr molybdate have also been carried out [14-16]. Crystallization and spherulitic growth of gadolinium, praseodymium, and mixed didymium heptamolybdate in silica gel medium [17, 18] have been investigated by many workers. Gadolinium molybdate $(\mathrm{GMO})$ with general formula $\mathrm{Gd}_{2}\left(\mathrm{MoO}_{4}\right)_{3}$ was observed to have luminescence properties [19]. Gofman et al. [19] studied radiation induced luminescence properties of gadolinium molybdate. Several other papers devoted to the luminescence properties of GMO doped with RE ions were also published [20-22]. Itoh [23] has studied luminescence properties of self-trapped exciton in $\mathrm{CdMoO}_{4}$. Keskar et al. [24] have carried out detailed study on thermal expansion of some other molybdates and tungstates such as $\mathrm{Gd}_{2} \mathrm{Mo}_{3} \mathrm{O}_{12}$ and $\mathrm{Gd}_{2} \mathrm{~W}_{3} \mathrm{O}_{12}$. Korah et al. [25] reported the growth and structural characteristics of gadolinium neodymium oxalate crystals. 
It is an established fact that an intrinsic material undergoes a change in its physical and chemical properties when it is grown in mixed form. Keeping in view the versatility of the gel technique in obtaining a large number of crystals, it was thought worthwhile to grow the mixed gadolinium calcium heptamolybdate (referred to as GdCaHM) crystal. In the present work, authors report the synthesis of GdCaHM crystals by controlled diffusion of chemical regents in silica gel medium. Literature survey reveals that no work has so far been reported on the synthesis and characterization of these crystals in sodium metasilicate gel at room temperature. Fourier transform infrared spectroscopy (FTIR) results suggest the presence of water of hydration in the grown crystal, so it was interesting to investigate in detail the thermal behaviour of these materials. Thus, this paper reports detailed results of synthesis, characterization, and thermal behavior of GdCaHM crystals grown by gel encapsulation technique.

\section{Experimental Technique}

2.1. Synthesis. The synthesis of gadolinium calcium heptamolybdate $(\mathrm{GdCaHM})$ is accomplished by allowing controlled diffusion of $\mathrm{Gd}^{3+}$ and $\mathrm{Ca}^{2+}$ ions in equal ratio through silica gel impregnated with the lower reactant providing molybdenum ions. The growth of mixed GdCaHM is achieved by using the system $\left(\mathrm{RCl}_{3}+\mathrm{R}^{/} \mathrm{Cl}_{2}\right)-\left(\mathrm{NH}_{4}\right)_{6} \mathrm{Mo}_{7} \mathrm{O}_{24}-\mathrm{NH}_{4} \mathrm{NO}_{3}-$ $\mathrm{HNO}_{3}-\mathrm{Na}_{2} \mathrm{SiO}_{3}$ (where $\mathrm{R}=\mathrm{Gd}^{3+}$ and $\mathrm{R}^{\prime}=\mathrm{Ca}^{2+}$ ) by using single gel single tube technique. The crystallization apparatus used for the growth consists of borosilicate glass tube of $20 \mathrm{~cm}$ length and $2.5 \mathrm{~cm}$ diameter. The high quality pure grade chemicals, from S. D. fine Chemical Ltd., used are gadolinium chloride (99\% A.R), calcium chloride (99\% A.R), ammonium molybdate (99\% A.R), ammonium nitrate (99\% A.R), and sodium metasilicate ( $99 \%$ A.R).

In the present case, silica gel is used as it is optically transparent and therefore crystals can be observed during the growth experiment. Furthermore, unlike other gels, the use of silica gel minimizes the effect due to precipitateprecipitate interaction and crystal impact on the wall of the container. Therefore, silica hydrogel is preferred and is the more common gel used for the growth experiment. It is obtained by the neutralization of sodium metasilicate. The gelling reaction is based on the hydrolytic process given by the following chemical reaction:

$$
\mathrm{Na}_{2} \mathrm{SiO}_{3}+3 \mathrm{H}_{2} \mathrm{O} \longrightarrow \mathrm{H}_{4} \mathrm{SiO}_{4}+2 \mathrm{NaOH}
$$

Silica gel was prepared by dissolving $212.14 \mathrm{~g}$ of sodium metasilicate $\left(\mathrm{Na}_{2} \mathrm{SiO}_{3}\right)$ in $1000 \mathrm{~mL}$ of distilled water so as to obtain gel solution of $1 \mathrm{M}$ concentration. The sodium metasilicate solution was left undisturbed for few days and clear solution was obtained on decantation. The second solution of lower reactant was prepared by adding ammonium molybdate and ammonium nitrate each weighing $817 \mathrm{mg}$ to $80 \mathrm{~mL}$ of distilled water. The solution was thoroughly mixed with the help of magnetic stirrer and then after about half an hour, $26 \mathrm{~mL}$ of concentrated $\mathrm{HNO}_{3}$ was added to it drop by drop till a white precipitate was formed. To this solution,
$20 \mathrm{~mL}$ of water was added so as to make a total volume of $250 \mathrm{~mL}$ of lower reactant having $0.005 \mathrm{M}$ concentration. The nitric acid content of the solution was added to adjust the desired $\mathrm{pH}$ value of the gel medium. The first solution (sodium metasilicate) was then mixed with the second (lower reactant-a source of molybdenum ions) in the crystallizer. The combined solution of desired $\mathrm{pH}$ was allowed to set in the crystallizer and then aged for desired time. Since in the present research work our objective is to grow mixed rare earth heptamolybdate crystal, therefore, upper reactant is a mixture of $\mathrm{GdCl}_{3}$ and $\mathrm{CaCl}_{2}$ in equal ratio. Upper reactant was prepared by dissolving $0.7 \mathrm{~g}$ of $\mathrm{GdCl}_{3}$ in $5 \mathrm{~mL}$ of $\mathrm{H}_{2} \mathrm{O}$ and $1.1 \mathrm{~g}$ of $\mathrm{CaCl}_{2}$ in $5 \mathrm{~mL}$ of $\mathrm{H}_{2} \mathrm{O}$. The two solutions were then mixed to get the required solution of $0.5 \mathrm{M}$ concentration in $1: 1$ ratio. This solution was then poured along the sides of the tube, ensuring that this process does not break the gel. The slow diffusion of upper reactant into the gel medium results into the reaction of rare earth/alkaline earth ion with heptamolybdate ion already present in the gel medium and subsequent formation of the proposed compound.

2.2. Characterization Technique. The different morphologies exhibited by the grown crystal were studied using an optical microscope (Epignost of Carl Zeiss Germany), whereas surface features of the grown crystals were studied using scanning electron microscopy (SEM, Model JEOL 840). The powder pattern was obtained using powder X-ray diffractometer (Rigaku Co. Ltd., Japan) with $\mathrm{Cu} K \alpha$ radiation $(\lambda=$ $1.54 \AA$ ) having scanning rate of $2^{\circ} / \mathrm{min}$. Energy dispersive Xrays analysis (EDAX) was recorded using dispersive spectrometer (INCA ENERGY EDAX) attached to the scanning electron microscope for carrying out elemental analysis of the grown crystals. Fourier transform infrared (FTIR) spectra were recorded in the wave number range of $4000-500 \mathrm{~cm}^{-1}$ on the Perkin-Elmer 781 spectrophotometer using KBr pellet method. The thermal behavior was investigated using thermogravimetric analysis (TGA), differential thermal analysis (DTA), and differential scanning calorimetry (DSC). TGA and DTA curves were recorded simultaneously by a thermal analyzer (Shimadzu make DTG-60) over the temperature range from 25 to $1000^{\circ} \mathrm{C}$ in the $\mathrm{N}_{2}$ atmosphere at a heating rate of $10^{\circ} \mathrm{C} / \mathrm{min}$ and flow rate of $30 \mathrm{~mL} / \mathrm{min}$. The DSC measurements were carried out on a DSC thermal analyzer (DSC-60 Shimadzu make) over a temperature range from 25 to $500^{\circ} \mathrm{C}$ in the nitrogen $\left(\mathrm{N}_{2}\right)$ atmosphere at a heating rate of $10^{\circ} \mathrm{C} / \mathrm{min}$ and flow rate of $30 \mathrm{~mL} / \mathrm{min}$.

\section{Results and Discussion}

3.1. Synthesis of Mixed Gd-Ca Heptamolybdate ( $\mathrm{GdCaHM).}$ In order to establish the optimum condition for the synthesis of GdCaHM in the form of single crystals of suitable size for scientific investigations, several experiments were performed under varying conditions of different growth parameters, namely, gel $\mathrm{pH}$, gel concentration, concentration of upper and lower reactant, and gel ageing. The optimum conditions for the growth of mixed GdCaHM crystal is achieved by using 


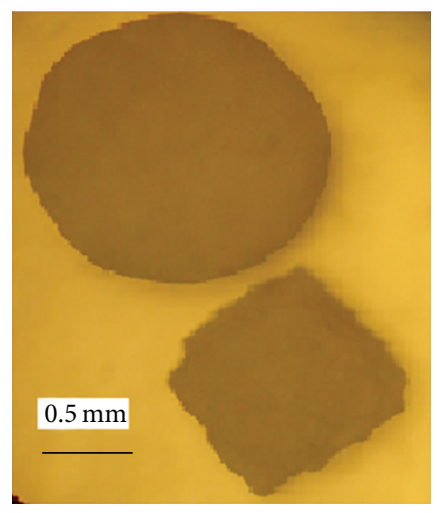

(a)

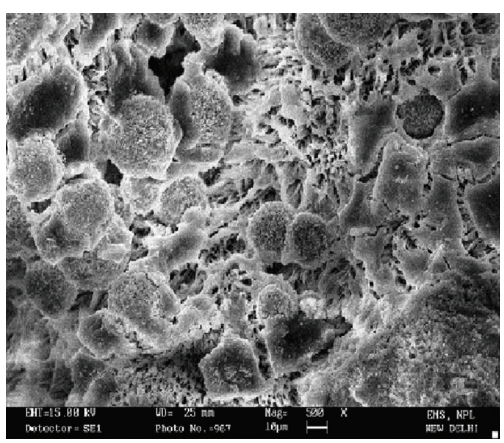

(b)

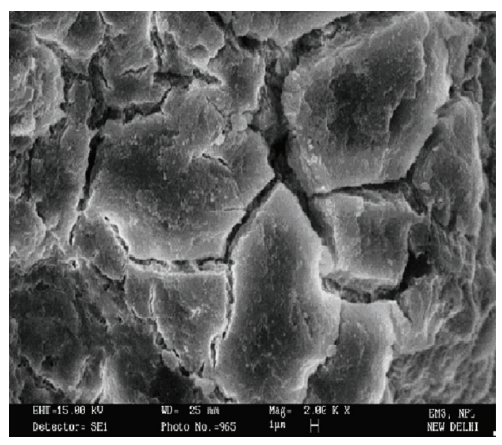

(c)

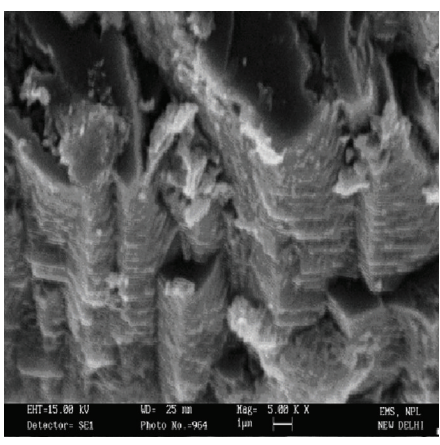

(d)

FIGURE 1: (a) Optical micrograph showing mixed Gd-Ca heptamolybdate (GdCaHM) crystal as a spherulite and a square platelet. (b) Scanning electron micrograph showing surface feature of spherulite crystals wherein number of spherulites are coalesced together with fiber like structure. (c) Scanning electron micrograph showing mixed GdCaHM crystal with cracks which results due to gold coating because of the presence of water content. (d) Scanning electron micrograph showing formation of growth layers in the growing crystal, suggesting twodimensional nucleation and pilling up of growth layers.

the following parameters: molarity of gel $\left(\mathrm{Na}_{2} \mathrm{SiO}_{3}\right): 0.5 \mathrm{M}$; molarity of lower reactant: $0.5 \mathrm{M}$; gel $\mathrm{pH}$ : 7.5 ; molarity of upper reactant: $0.5 \mathrm{M}$; and gelation period: $48 \mathrm{~h}$.

3.2. Optical and Scanning Electron Microscopy. Mixed GdCa heptamolybdate (GdCaHM) crystals grown by single gel single tube technique assume various types of morphologies, which include spherulites, multifaceted, and square platelets. Figure 1(a) is an optical micrograph showing the grown crystal as spherulite and square platelet. These spherulites are in fact an agglomeration of number of tiny crystals. In order to study the surface features, scanning electron microscopic study was performed on the grown crystal. Figure 1(b) is an electron micrograph of GdCaHM showing surface features of the spherulite. Here, one can see number of small spherulites along with some fiber like structure. This fiber like structure can be due to the adhering of the silica gel. This adhering of the silica gel on some of the spherulite crystals is because of not being properly cleaned while taking crystal out of the crystallizer. Figure 1(c) shows single crystal of GdCaHM with cracks. These cracks do not have specific crystallographic direction and results when the crystals were coated with gold in the vacuum coating plant and examined under scanning electron microscope. Such type of cracking is often associated with crystal having water of crystallization [13, 26, 27]. Figure 1(d) shows an interesting feature on one of the mixed Gd-Ca crystals. On close examination, it reveals growth layers suggesting two-dimensional spreading and piling up of growth layers, which is a consequence of preferred nucleation at the corners and at the edges of the face of growing crystal at relatively high supersaturation.

3.3. X-Ray Diffraction Analysis (XRD). The powder X-ray diffractogram of GdCaHM crystal is shown in Figure 2. The crystallinity is quite clear from diffractogram because of the occurrence of sharp peaks at specific $2 \theta$ Bragg angles. The details of the XRD plot depicting " $d$ " spacing and corresponding [hkl] planes are given in Table 1.

3.4. Energy Dispersive X-Ray Analysis (EDAX). To study the elemental composition, qualitative and quantitative analysis was performed by energy dispersive $\mathrm{X}$-ray analysis (EDAX). Figure 3 shows the EDAX pattern of the grown crystal. The EDAX pattern shows the peaks corresponding to $\mathrm{Gd}, \mathrm{Ca}$, $\mathrm{Mo}, \mathrm{O}$, and $\mathrm{Si}$ which suggest the presence of almost all the constituents expected to be present in the material. The 


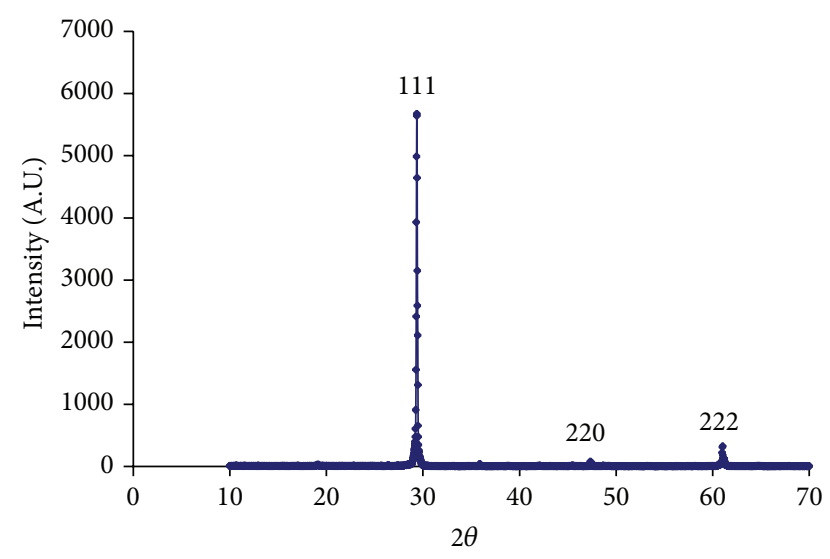

FIGURE 2: X-ray diffractogram of mixed GdCaHM showing various [hkl] planes.

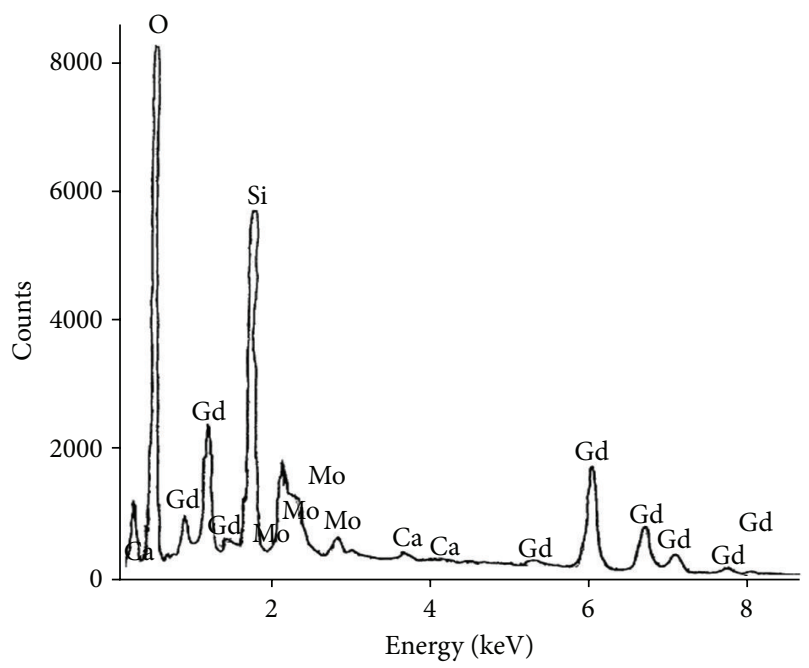

Figure 3: Energy dispersive X-ray analysis (EDAX) revealing the presence of major elements in the mixed GdCaHM crystal along with the presence of silica $(\mathrm{Si})$.

TABLE 1: Compiled data of various hkl planes corresponding to different Bragg angle and inter planer spacing for mixed $\mathrm{GdCa}$ heptamolybdate (GdCaHM).

\begin{tabular}{lccc}
\hline$d$-spacing $(\AA)$ & 2 theta $(\theta)$ & {$[$ hkl] planes } & $\begin{array}{c}\text { Intensity } \\
\text { (counts) }\end{array}$ \\
\hline 3.14 & 29.38 & 111 & 6173 \\
2.10 & 47.46 & 220 & 559 \\
1.77 & 61.04 & 222 & $\mathbf{8 2 3}$ \\
\hline
\end{tabular}

presence of $\mathrm{Si}$ is attributed to the adhering of silica gel because of not being properly cleaned as has also been seen and discussed in Figure 1(b). Quantitative estimation of elements present in the crystal through its weight and atomic percentage based on the proposed composition $\mathrm{GdCaMo}_{7} \mathrm{O}_{24} \cdot 8 \mathrm{H}_{2} \mathrm{O}$ is given in Table 2. The theoretical (quantitative) values based on the proposed composition are in good agreement with experimental (qualitative) values; thus, based on this analysis,
TABLE 2: Experimental and theoretical calculated composition obtained from energy dispersive X-ray analysis (EDAX) of various constituent elements present in case of $\mathrm{GdCa}$ heptamolybdate $(\mathrm{GdCaHM})$ crystal for composition of $\mathrm{GdCaMo}_{7} \mathrm{O}_{24} \cdot 8 \mathrm{H}_{2} \mathrm{O}$.

\begin{tabular}{lcccc}
\hline \multirow{2}{*}{ Element } & \multicolumn{2}{c}{ Experimental values } & \multicolumn{2}{c}{ Theoretical values } \\
& Wt. (\%) & At. (\%) & Wt. (\%) & At. (\%) \\
\hline Oxygen & 36.87 & 77.57 & 37.06 & 78.04 \\
Calcium & 3.38 & 2.71 & 2.9 & 2.43 \\
Molybdenum & 47.5 & 17 & 48.65 & 17.07 \\
Gadolinium & 12.25 & 2.71 & 11.3 & 2.43 \\
\hline
\end{tabular}

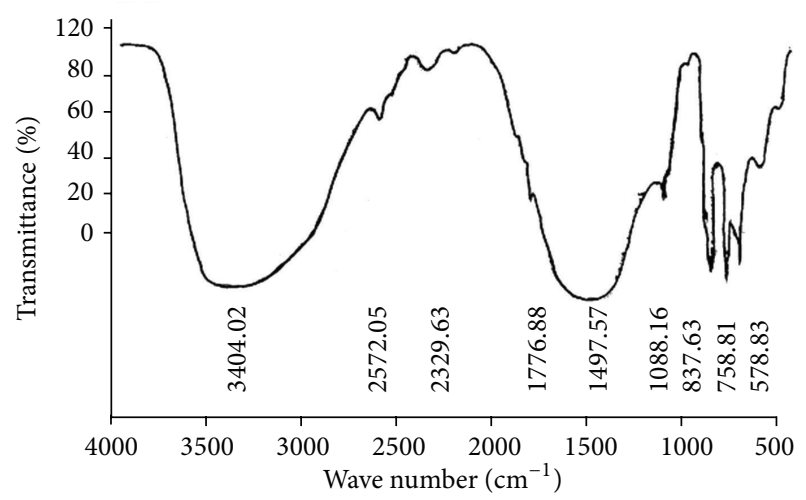

FIGURE 4: FTIR spectrum of the crystal depicting the various functional groups present in the mixed GdCaHM crystal.

it is clear that these crystals belong to the heptamolybdate series with the composition $\mathrm{GdCaMo}_{7} \mathrm{O}_{24} \cdot 8 \mathrm{H}_{2} \mathrm{O}$. The water of hydration associated with this system is revealed by FTIR spectrum as given in the next section. The proposed composition was further verified by the thermal analysis given in Section 3.6 (TGA, DTG, and DTA) which support the inference that the material is associated with water of hydration, which dissociates from the material at different stages of decomposition occurring at different temperature intervals.

3.5. Fourier Transform Infrared Spectroscopy (FTIR). Figure 4 shows an infrared spectrum for GdCaHM crystals recorded by using $\mathrm{KBr}$ pellet technique, for wave numbers in the range from 500 to $4000 \mathrm{~cm}^{-1}$. As seen from the spectrum, there is a broad and strong peak at $3404.2 \mathrm{~cm}^{-1}$ which is due to water and strong stretching modes of the $\mathrm{OH}^{-}$group (water symmetry structure). The weak and broad peak observed at $2329.63 \mathrm{~cm}^{-1}$ can be attributed to the presence of $\mathrm{Si}-\mathrm{H}$ bond. EDAX analysis also confirms the presence of $\mathrm{Si}$ in the material. The strong and broad peak at $1497.57 \mathrm{~cm}^{-1}$ is indicative of vibration of oxygen ion for $\mathrm{Gd}$. The strong, sharp peak observed at $837.63 \mathrm{~cm}^{-1}$ is due to symmetric or antisymmetric stretching vibrations of the heptamolybdate ion $\left(\mathrm{Mo}_{7} \mathrm{O}_{24}\right)$ [28-30]. The peaks occurring at 758.81 and $578.83 \mathrm{~cm}^{-1}$ are attributed to the presence of metal oxygen bonds. A comparative assignment of prominent peaks of FTIR spectra is given in the Table 3 . 


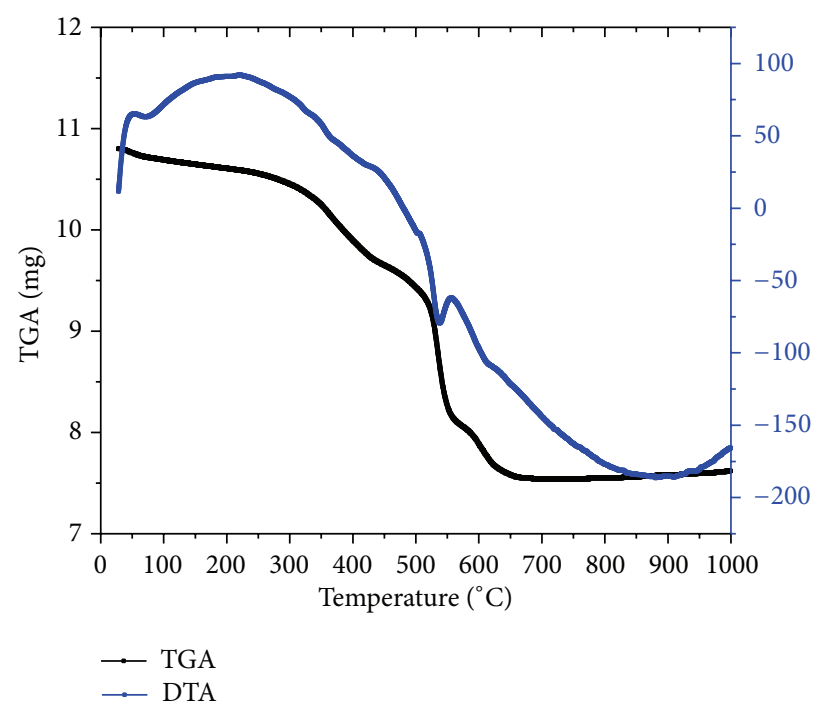

(a)

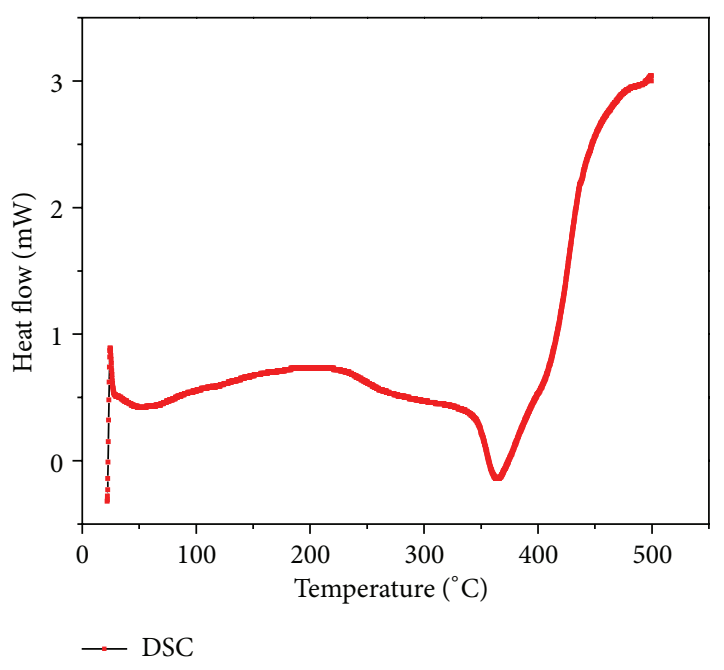

(b)

FIGURE 5: (a) Thermal analysis curve showing simultaneous recording of TGA, DTG, and DTA curves for mixed GdCaHM crystal. (b) DSC plot of mixed GdCaHM crystal.

TABLE 3: Infrared band/peak assignments of some selected bands/ peaks for mixed GdCa heptamolybdate (GdCaHM) crystal.

\begin{tabular}{lc}
\hline IR band $/ \mathrm{cm}^{-1}$ & Assignments of bands/peaks \\
\hline 3404.2 & Water symmetry structure \\
2329.63 & Presence of $\mathrm{Si}-\mathrm{H}$ bond \\
1776.63 & Attributed to water bending \\
1497.57 & Vibration of oxygen ion for gadolinium \\
837.63 & Vibrations of molybdate ions $\left(\mathrm{Mo}_{7} \mathrm{O}_{24}\right)$ \\
578.83 & Presence of metal oxygen bond \\
\hline
\end{tabular}

3.6. Thermal Analysis. Thermal analysis is a very useful technique in order to assess the thermal stability of a material. Thermal behavior of GdCaHM has been studied by using various thermoanalytical techniques which include thermogravimetry (TG), differential thermal analysis (DTA), and differential scanning calorimetry (DSC). Figure 5(a) shows the thermograph where in TG and DTA curves are simultaneously recorded. The recorded thermograph (Figure 5(a)) was first analyzed to obtain information about the percentage of mass loss at different temperatures and hence about crystal stability. From the curve, it is clear that when the crystal is heated at a uniform rate of $10^{\circ} \mathrm{C} \mathrm{min}^{-1}$, its mass is found to be lost continuously as a function of applied temperature. The thermal decomposition takes place in three different stages starting from 28 to $666^{\circ} \mathrm{C}$.

The first stage of decomposition begins at a temperature of $28^{\circ} \mathrm{C}$ and get completed at $440^{\circ} \mathrm{C}$, leading to weight loss of $10.38 \%$ (Table 4 ). The weight loss in the first stage of decomposition is attributed to the loss of eight water $\left(\mathrm{H}_{2} \mathrm{O}\right)$ molecules from the grown composition. The calculated loss of eight water molecules $\left(8 \mathrm{H}_{2} \mathrm{O}\right)$ comes out to be $10.31 \%$ which is close approximation to the observed values, thereby confirming the results from EDAX analysis that the composition consists of eight water molecules with it. The second stage of decomposition starts at $441^{\circ} \mathrm{C}$ which continuous up to $543^{\circ} \mathrm{C}$. Up to the end of this stage, a weight loss of $11.49 \%$ is observed. The weight loss in the second stage corresponds to the removal of one molybdenum $\left(\mathrm{MoO}_{3}\right)$ molecule. The third decomposition stage begins at $544^{\circ} \mathrm{C}$ and continuous up to $666^{\circ} \mathrm{C}$ leading to weight loss of $6.49 \%$. The weight loss in the third stage is due to the loss of one calcium peroxide $\left(\mathrm{CaO}_{2}\right)$ molecule. The end product obtained after the third stage of decomposition is anhydrous gadolinium hexamolybdate $\left(\mathrm{GdMo}_{6} \mathrm{O}_{19}\right)$.

From the TG curve, which is reported up to a temperature of $1000^{\circ} \mathrm{C}$, it is clear that material remains stable after the third stage of decomposition is completed. Table 4 gives the summary of results obtained from the thermal decomposition for different temperature ranges with composition as $\mathrm{GdCaMo}_{7} \mathrm{O}_{24} \cdot 8 \mathrm{H}_{2} \mathrm{O}$. It can be seen that the calculated weight losses are in close proximity with the observed values. DTA curve shows an exothermic peak at $225^{\circ} \mathrm{C}$, which corresponds to the first stage of decomposition of TG curve. There is wellmarked endothermic peak in DTA curve at $535^{\circ} \mathrm{C}$, which corresponds to the second stage of decomposition of TG curve. The third peak (exothermic) occurs at $565^{\circ} \mathrm{C}$, which corresponds to the third stage of decomposition of TG curve. Figure 5(b) is a representative DSC plot of GdCaHM crystals. From the DSC plot, one can see an endothermic peak to which corresponding enthalpy change is about $19.97 \mathrm{~mJ}$. The second endothermic peak in the DSC plot is at a temperature of $365^{\circ} \mathrm{C}$ thereby suggesting that there is a mass loss in the material which is in quite good agreement with the mass loss in the TGA curve. The enthalpy involved in this endothermic peak is $-96.68 \mathrm{~mJ}$. 
TABLE 4: Results of thermal decomposition for different temperature ranges with observed and calculated weight loss for mixed crystal with composition $\mathrm{GdCaMo}_{7} \mathrm{O}_{24} \cdot 8 \mathrm{H}_{2} \mathrm{O}$.

\begin{tabular}{lccrr}
\hline Stage & Temp. $\left({ }^{\circ} \mathrm{C}\right)$ & Decomposition step & \multicolumn{2}{c}{ Weight loss (\%) } \\
Ist & $28-440$ & $\mathrm{GdCaMo}_{7} \mathrm{O}_{24} \cdot 8 \mathrm{H}_{2} \mathrm{O} \rightarrow \mathrm{GdCaMo}_{7} \mathrm{O}_{24}+8 \mathrm{H}_{2} \mathrm{O}$ & 10.38 & 10.31 \\
IInd & $441-543$ & $\mathrm{GdCaMo}_{7} \mathrm{O}_{24} \rightarrow \mathrm{GdCaMo}_{6} \mathrm{O}_{21}+\mathrm{MoO}_{3}$ & 11.41 & 11.49 \\
IIIrd & $544-666$ & $\mathrm{GdCaMo}_{6} \mathrm{O}_{21} \rightarrow \mathrm{GdMo}_{6} \mathrm{O}_{19}+\mathrm{CaO}_{2}$ & 7.77 & 6.49 \\
\hline
\end{tabular}

TABLE 5: Various kinetic parameters calculated from the TGA using three different relations.

\begin{tabular}{|c|c|c|c|c|c|}
\hline STAGE & TG method & Order of reaction $(n)$ & $\begin{array}{l}\text { Activation energy } \\
\left(\mathrm{K} \mathrm{Jmol}^{-1}\right)\end{array}$ & $\begin{array}{c}\text { Frequency factor } Z \\
\left(\mathrm{~S}^{-1}\right)\end{array}$ & $\begin{array}{c}\text { Entropy } \Delta S^{*} \\
\left(\mathrm{JK}^{-1} \mathrm{~mol}^{-1}\right)\end{array}$ \\
\hline \multirow{3}{*}{ First } & Horowitz-Metzger & $n=1 / 2$ & 22.3 & 1.19 & -247 \\
\hline & Coats-Redfern & $n=1 / 2$ & 25.7 & $2 \times 10^{-4}$ & -259.75 \\
\hline & Piloyan-Novikova & nil & 9.514 & $4.4 \times 10$ & -294.3 \\
\hline \multirow{3}{*}{ Second } & Horowitz-Metzger & $n=1 / 2$ & 152.31 & $1.0 \times 10^{8}$ & -99.46 \\
\hline & Coats-Redfern & $n=1 / 2$ & 111.86 & $1.6 \times 10^{5}$ & -153.11 \\
\hline & Piloyan-Novikova & nil & 89.22 & $1.1 \times 10$ & -328.68 \\
\hline \multirow{3}{*}{ Third } & Horowitz-Metzger & $n=1 / 2$ & 100.85 & $1.6 \times 10^{4}$ & -172.67 \\
\hline & Coats-Redfern & $n=1 / 2$ & 87.05 & $2.3 \times 10^{-5}$ & -341.97 \\
\hline & Piloyan-Novikova & nil & 38.12 & $9 \times 10^{-2}$ & -273.53 \\
\hline
\end{tabular}

3.7. Solid State Kinetic Parameters of Thermal Decomposition Analysis. For studying the kinetics of solid state decomposition, three equations, namely, Horowitz-Metzger [31], Coats-Redfern [32], and Piloyan-Novikova [33], were used for calculating various kinetic parameters such as activation energy $\left(E_{a}\right)$, order of reaction $(n)$, frequency factor $(Z)$, and entropy $\left(\Delta S^{*}\right)$. The kinetics was studied by nonisothermal methods in the temperature range of $28-666^{\circ} \mathrm{C}$. As the decomposition in the constituent element takes place in three different stages (Figure 5(a)), so all the three stages of decomposition were used for calculating various kinetic parameters.

In case of Horowitz-Metzger relation [31], a graph was plotted between $\log [g(\alpha)]$ versus $\theta$ for different values of $n$ (order of reaction) $=1,1 / 2,1 / 3,2 / 3$. Figure $6(\mathrm{a})$ shows the best linear fit for $n=1 / 2$, that is, for $g(\alpha)=2\left[1(1-\alpha)^{1 / 2}\right]$. Since the value of order of reaction " $n$ " comes out to be $1 / 2$, thereby suggesting that the mechanism of decomposition is based on contracting cylindrical model. In this model, the nucleation occurs rapidly on the decomposing nuclei and the rate of degradation is controlled by the resulting reaction interface, which progress towards the center of the crystal. The derivation of the mathematical model for contracting cylindrical decomposition depends on the crystal shape. This decomposition model has also been observed in case of pure and cadmium doped barium phosphate single crystals [34]. Dehydration of calcium oxalate monohydrate also follows geometrical contraction model [35]. The activation energy $\left(E_{a}\right)$ was calculated from the slope of the graph between $\log [g(\alpha)]$ versus $\theta$ which for first stage comes out to be $22.3 \mathrm{KJmol}^{-1}$. The value of the frequency factor $(Z)$ and entropy $\left(\Delta S^{*}\right)$ was also calculated using the various relations [34] which comes out to be $1.19 \mathrm{~S}^{-1}$ and $-247 \mathrm{JK}^{-1} \mathrm{~mol}^{-1}$, respectively, for first stage of decomposition. Similarly, it has been observed that on applying Horowitz-Metzger relation, the value of order of reaction $(n)$ comes out to be $1 / 2$ for second and third stage of decomposition thereby suggesting that for all the three stages of decomposition contracting cylindrical model is applicable. Figure 6(a) shows the best linear fit for all the three stages of decomposition. The energy of activation $\left(E_{a}\right)$, frequency factor $(Z)$, and entropy $\left(\Delta S^{*}\right)$ were also calculated for second and third stages of decomposition, the values of which are given in Table 5.

Coats-Redfern relation [32] was also applied to find the mechanism of decomposition for three different stages. Figure 6(b) shows graph of $\log \left[g(\alpha) / T^{2}\right]$ versus $1 / T$ for $n=$ $1 / 2$, where $g(\alpha)=2\left[1(1-\alpha)^{1 / 2}\right]$. The order of reaction (n) from Coats-Redfern relation comes out to be $1 / 2$, which again confirms that decomposition proceeds according to contracting cylindrical model as has been observed in case of Horowitz-Metzger relation. The energy of activation $\left(E_{a}\right)$, frequency factor $(Z)$, and entropy $\left(\Delta S^{*}\right)$ were calculated using appropriate equations for all the three stages of decomposition and the values are shown in Table 5.

Piloyan-Novikova relation [33] does not give the value of order of reaction and hence no model for decomposition. However, from this relation by plotting a graph between $\log \left[(\alpha) / T^{2}\right]$ versus $1 / T$, one can calculate the value of activation energy $\left(E_{a}\right)$ and frequency factor $(Z)$ from the slope and intercept, respectively. 


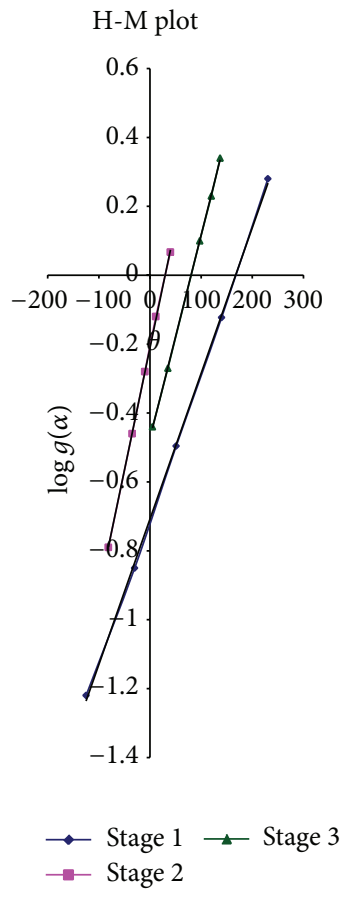

(a)

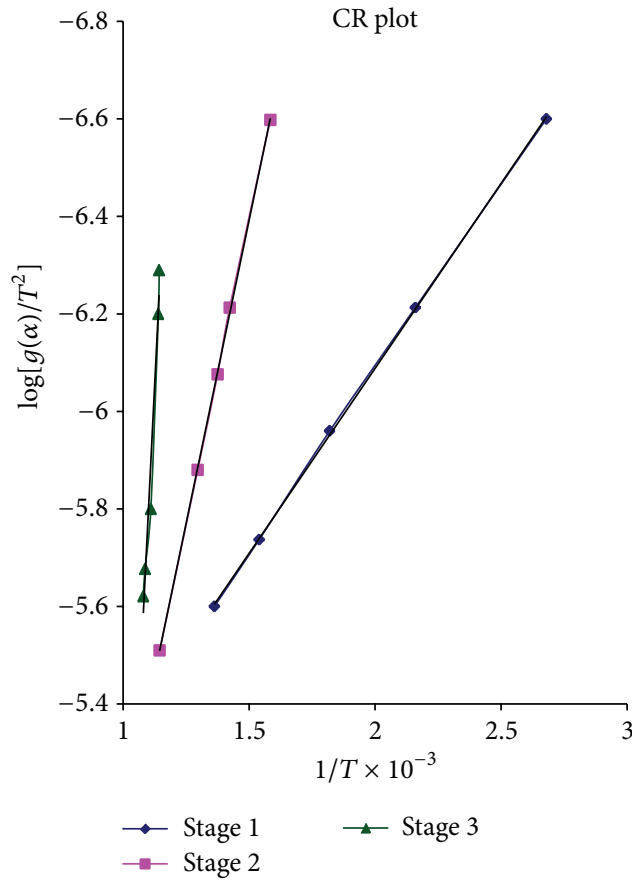

(b)

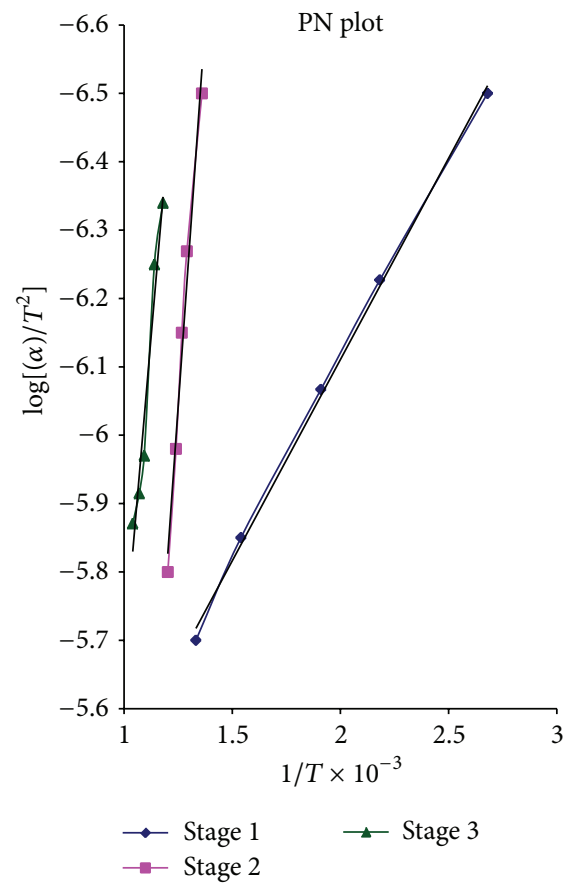

(c)

Figure 6: (a) Horowitz-Metzger plot of GdCaHM for three stages of decomposition. (b) Coats-Redfern plot of GdCaHM for three stages of decomposition. (c) Piloyan-Novikova plot of GdCaHM for three stages of decomposition.

Table 5 thus gives compiled data for various kinetic parameters, that is, energy of activation $\left(E_{a}\right)$, order of reaction $(n)$, frequency factor $(Z)$, and entropy $\left(\Delta S^{*}\right)$ obtained on applying the equations of Horowitz-Metzger, Coat-Redfern, and Piloyan-Novikova relations for mixed GdCaHM crystal.
From these kinetic parameters and from all the three different stages of decomposition, we conclude that in case of mixed GdCaHM crystal, the maximum activation energy $\left(E_{a}\right)$ is required for second stage of decomposition because in this stage molybdenum trioxide $\left(\mathrm{MoO}_{3}\right)$ decomposes from the 
system whereas minimum activation energy is required for the first stage of decomposition because in this stage water $\left(\mathrm{H}_{2} \mathrm{O}\right)$ is decomposed.

\section{Conclusions}

From the results obtained on mixed gadolinium calcium heptamolybdate (GdCaHM) crystals one can draw the following broad conclusions.

(i) Crystals of GdCaHM having composition $\mathrm{GdCaMo}_{7} \mathrm{O}_{24} \cdot 8 \mathrm{H}_{2} \mathrm{O}$ have been successfully synthesized by single gel single tube technique.

(ii) The optical and scanning electron microscopic studies reveal growth of mixed GdCaHM crystals, which exhibit various morphologies. Thus, mixed GdCaHM crystal grows as single crystal with square platelet as well as spherulites.

(iii) The qualitative and quantitative elemental analysis, employing energy dispersive X-ray analysis, confirms the growth of mixed GdCaHM crystals. The calculated and experimental weight percentage for different elements is in good agreement for the composition $\mathrm{GdCaMo}_{7} \mathrm{O}_{24} \cdot 8 \mathrm{H}_{2} \mathrm{O}$.

(iv) The FTIR spectroscopy establishes the crystal to be hydrated with all functional groups expected to be present in the crystal.

(v) GdCaHM crystals are stable up to a temperature of $28^{\circ} \mathrm{C}$ and then starts decomposing and this decomposition process continues up to $666^{\circ} \mathrm{C}$. The whole decomposition process is completed in three different stages of decomposition.

(vi) DTA and DSC curves indicate that there may be some physical transformations (besides mass changes associated with loss of water) in case of mixed GdCaHM crystal.

(vii) By using Horowitz-Metzger and Coats-Redfern equations for all the three different stages of decomposition, the best linear fit is obtained for an order of reaction $n=1 / 2$. This indicates that the decomposition proceeds according to the contracting cylindrical kinetic model in GdCaHM crystal.

(viii) The various kinetic parameters like energy of activation $\left(E_{a}\right)$, frequency factor $(Z)$, and entropy of decomposition $\left(\Delta S^{*}\right)$ using three different equations were calculated for all the three stages of decomposition, indicating that minimum activation energy is required to break the molecules in the first stage of decomposition because the water molecules are loosely bound to the mixed crystal.

\section{Conflict of Interests}

The authors declare that there is no conflict of interests regarding the publication of this paper.

\section{References}

[1] H. J. Borchordt and P. E. Bierstedt, "A ferroelectric laser host," Applied Physics Letters, vol. 8, pp. 50-52, 1966.

[2] J. R. Barkley, L. H. Brixner, and E. M. Horgan, in Proceedings of the IEEE Symposium on Applications of Ferroelectrics, York Town Heights, NY, USA, 1971.

[3] J. R. Barkley, J. R, L. H. Brixner, E. M. Horgan, and R. K. Waring, "Control and application of domain wall motion in gadolinium molybdate," Ferroelectrics, vol. 3, pp. 191-197, 1972.

[4] J. Sapriel and R. Vacher, "Photoelastic tensor components of $\mathrm{Gd}_{2}\left(\mathrm{MoO}_{4}\right)$," Journal of Applied Physics, vol. 48, no. 3, pp. 1191$1194,1977$.

[5] L. H. Brixner, "Single crystal growth of the $\mathrm{Ln}_{2}\left(\mathrm{MoO}_{4}\right)_{3}$-type rare earth molybdates," Journal of Crystal Growth, vol. 18, no. 3, pp. 297-302, 1973.

[6] H. K. Henisch, Crystal Growth in Gels, Pennsylvania State University Press, University Park, Pa, USA, 1970.

[7] H. K. Hektisch, J. Dennis, and J. I. Hanoka, "Crystal growth in gels," Journal of Physics and Chemistry of Solids, vol. 26, no. 3, pp. 493-496, 1965.

[8] J. Isac and M. A. Ittyachen, "Growth and characterization of rare-earth mixed single crystals of samarium barium molybdate," Bulletin of Materials Science, vol. 15, no. 4, pp. 349-353, 1992.

[9] S. Bhat and P. N. Kotru, "Growth of lanthanum heptamolybdate crystals by gel technique," Crystal Research and Technology, vol. 29, no. 3, pp. 325-333, 1994.

[10] S. Bhat, P. N. Kotru, and M. L. Koul, "Growth of neodymium heptamolybdate crystals in silica gels at ambient temperatures," Materials Science and Engineering B, vol. 23, no. 2, pp. 73-82, 1994.

[11] S. Bhat, M. L. Koul, and P. N. Kotru, "Growth of neodymium lanthanum heptamolybdate mixed crystals in silica gels," Materials Science and Engineering B, vol. 34, no. 2-3, pp. 138-146, 1995.

[12] S. Brat, S. K. Khosa, P. N. Kotru, and R. P. Tandon, "Dielectric characteristics of gel-grown mixed neodymium-lanthanumheptamolybdate crystals," Journal of Materials Science Letters, vol. 14 , no. 8, pp. 564-567, 1995.

[13] S. Bhat and P. N. Kotru, "Characterization of lanthanum heptamolybdate crystals grown from silica gels," Materials Chemistry and Physics, vol. 39, no. 2, pp. 118-123, 1994.

[14] V. Hangloo, S. Pandita, K. K. Bamzai, P. N. Kotru, and N. Sahni, "Growth and characterization of pure Gd-heptamolybdate and mixed Gd-Ba-molybdate crystals," Crystal Growth and Design, vol. 3, no. 5, pp. 753-759, 2003.

[15] V. Hangloo, K. K. Bamzai, P. N. Kotru, and M. L. Koul, “Thermal characteristics of pure and substituted gel grown Gd-molybdate crystals," Bulletin of Materials Science, vol. 27, no. 5, pp. 395-401, 2004.

[16] V. Hangloo, K. K. Bamzai, P. N. Kotru, N. Sahni, and R. Dhar, "Growth, characterization and thermal behaviour of gel grown mixed Gd-Sr-molybdate crystals," Materials Science and Engineering A, vol. 358, no. 1-2, pp. 334-342, 2003.

[17] S. Pandita, R. Tickoo, K. K. Bamzai, P. N. Kotru, and N. Sahni, "Crystallization of mixed rare earth (didymium) molybdates in silica gel," Bulletin of Materials Science, vol. 24, no. 5, pp. 435440, 2001.

[18] V. Hangloo, S. Pandita, K. K. Bamzai, P. N. Kotru, and N. Sahni, "Spherulitic growth of single (Gd \& Pr) and mixed Di rare earth 
heptamolybdates in silica gels," Journal of Materials Science, vol. 39, no. 5, pp. 1743-1749, 2004.

[19] I. A. Gofman, V. A. Pustovarov, N. I. Lobachevskaya, and V. D. Zhuravlev, "Intrinsic and radiation-induced defect luminescence of gadolinium molybdate under UV- and VUVexcitation," Radiation Measurements, vol. 56, pp. 44-48, 2013.

[20] C. Guo, T. Chen, L. Luan, W. Zhang, and D. Huang, "Luminescent properties of $\mathrm{R}_{2}\left(\mathrm{MoO}_{4}\right)_{3}: \mathrm{Eu}^{3+}(\mathrm{R}=\mathrm{La}, \mathrm{Y}, \mathrm{Gd})$ phosphors prepared by sol-gel process," Journal of Physics and Chemistry of Solids, vol. 69, no. 8, pp. 1905-1911, 2008.

[21] X. He, J. Zhou, N. Lian, J. Sun, and M. Guan, "Sm ${ }^{3+}$-activated gadolinium molybdate: an intense red-emitting phosphor for solid-state lighting based on InGaN LEDs," Journal of Luminescence, vol. 130, no. 5, pp. 743-747, 2010.

[22] Y. Pan, Q. Zhang, C. Zhao, and Z. Jiang, "Luminescent properties of novel $\mathrm{Ho}^{3+}$ and $\mathrm{Tm}^{3+}$ doped gadolinium molybdate nanocrystals synthesized by the Pechini method," Solid State Communications, vol. 142, no. 1-2, pp. 24-27, 2007.

[23] M. Itoh, "Luminescence study of self-trapped excitons in $\mathrm{CdMoO}_{4}$," Journal of Luminescence, vol. 132, no. 3, pp. 645-651, 2012.

[24] M. Keskar, N. D. Dahale, K. Krishnan, and N. K. Kulkarni, "Thermal expansion studies of $\mathrm{Gd}_{2} \mathrm{Mo}_{3} \mathrm{O}_{12}$ and $\mathrm{Gd}_{2} \mathrm{~W}_{3} \mathrm{O}_{12}$," Materials Research Bulletin, vol. 44, no. 4, pp. 901-905, 2009.

[25] I. Korah, C. Joseph, and M. A. Ittyachen, "Growth and structural characterization of gadolinium neodymium oxalate crystals grown in hydro-silica gel," Journal of Minerals and Materials Characterization and Engineering, vol. 9, no. 12, pp. 1081-1086, 2010.

[26] S. Bhat and P. N. Kotru, "Characterization of gel-grown neodymium heptamolybdate crystals," Journal of Materials Science, vol. 30, no. 10, pp. 2694-2700, 1995.

[27] P. N. Kotru, K. K. Raina, and M. L. Koul, "Characterization of lanthanum heptamolybdate crystals grown from silica gel," Journal of Materials Science, vol. 21, pp. 3933-3940, 1986.

[28] K. Nakamoti John Wiley \& Sons, New York, NY, USA, 3rd edition, 1978.

[29] R. H. Busey and O. L. Keller, "Structure of the aqueous pertechnetate ion by Raman and infra red Spectroscopy Raman and Infra red spectra of crystalline $\mathrm{KTcO}_{4}, \mathrm{KRe}_{4}, \mathrm{Na}_{2} \mathrm{MoO}_{4}$ $\mathrm{Na}_{2} \mathrm{WO}_{4} \mathrm{Na}_{2} \mathrm{MoO}_{4} \cdot 2 \mathrm{H}_{2} \mathrm{O}$ and $\mathrm{Na}_{2} \mathrm{WO}_{4} \cdot 2 \mathrm{H}_{2} 0$," The Journal of Chemical Physics, vol. 41, pp. 215-225, 1964.

[30] G. M. Clark and W. P. Doyle, "Infra-red spectra of anhydrous molybdates and tungstates," Spectrochimica Acta, vol. 22, no. 8, pp. 1441-1447, 1966.

[31] H. H. Horowitz and G. Metzger, "A new analysis of thermogravimetric traces," Analytical Chemistry, vol. 35, no. 10, pp. 1464$1468,1963$.

[32] A. W. Coats and J. P. Redfern, "Kinetic parameters from thermogravimetric data," Nature, vol. 201, no. 4914, pp. 68-69, 1964.

[33] G. O. Piloyan, I. D. Ryabchikov, and O. S. Novikova, "Determination of activation energies of chemical reactions by differential thermal analysis," Nature, vol. 212, no. 5067, p. 1229, 1966.

[34] S. Suri, K. K. Bamzai, and V. Singh, "Growth and thermal kinetics of pure and cadmium doped barium phosphate single crystal," Journal of Thermal Analysis and Calorimetry, vol. 105, no. 1, pp. 229-238, 2011.

[35] Z. M. Gao, I. Amasaki, and M. Nakada, "A description of kinetics of thermal decomposition of calcium oxalate monohydrate by means of the accommodated Rn model," Thermochimica Acta, vol. 385, no. 1-2, pp. 95-103, 2002. 

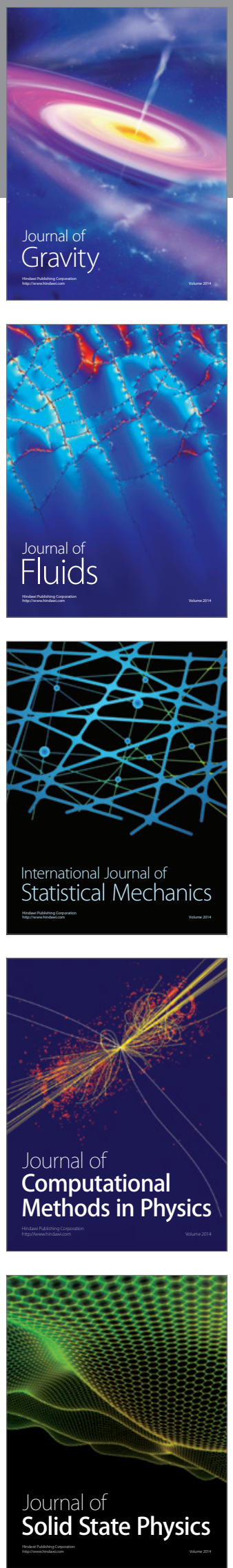

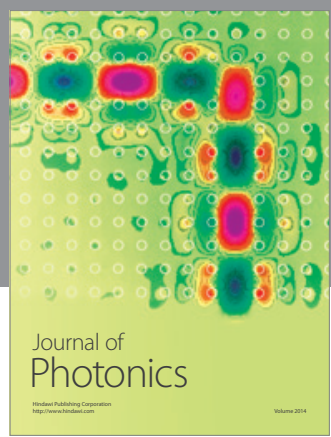

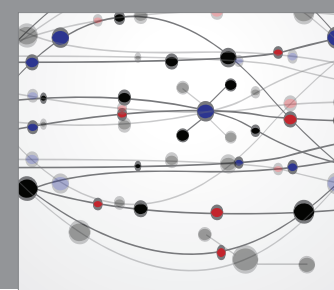

The Scientific World Journal

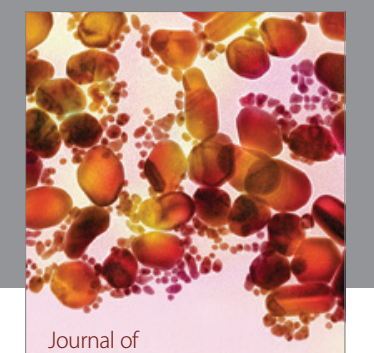

Soft Matter
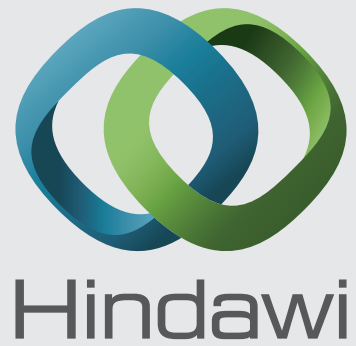

Submit your manuscripts at

http://www.hindawi.com
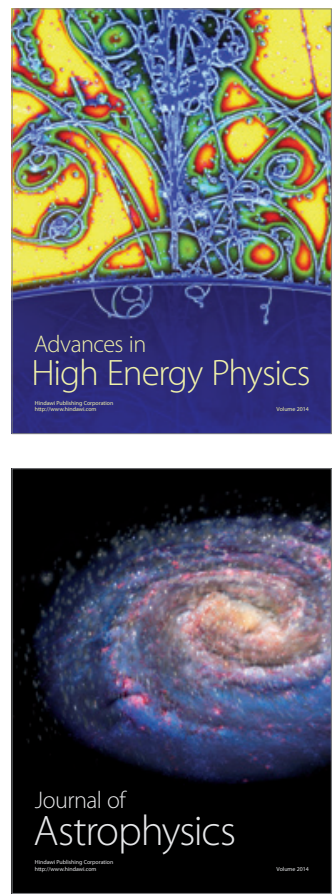
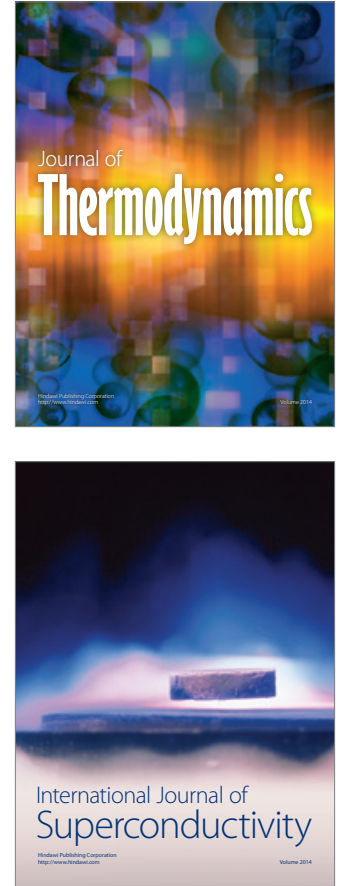
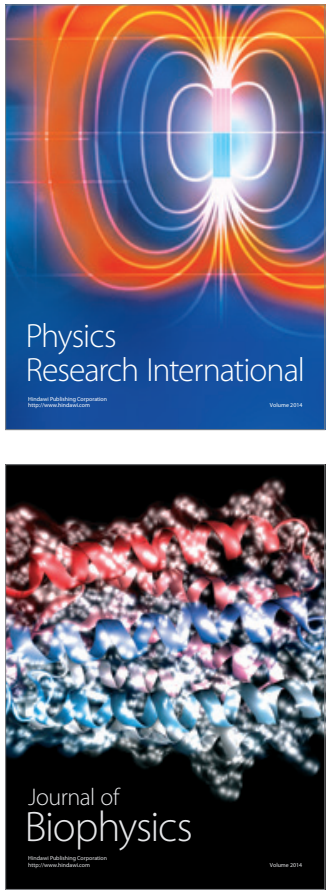
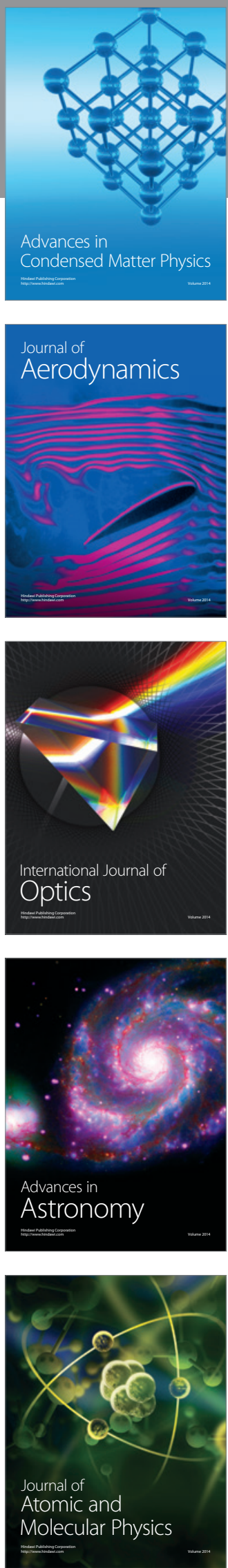